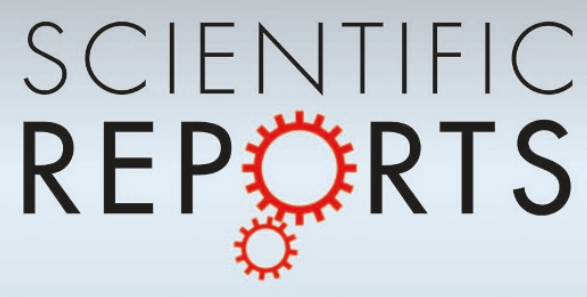

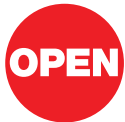

SUBJECT AREAS: APPLIED PHYSICS

MECHANICAL ENGINEERING

FLUID DYNAMICS

MECHANICAL PROPERTIES

Received

21 January 2013

Accepted

4 April 2013

Published

25 April 2013

Correspondence and requests for materials should be addressed to W.L.Z. (weili.zhang@ okstate.edu) or X.Z. (xiang@berkeley.edu)

\section{Acoustic rainbow trapping}

\author{
Jie Zhu', Yongyao Chen ${ }^{2}$, Xuefeng Zhu ${ }^{1,3}$, Francisco J. Garcia-Vidal ${ }^{4}$, Xiaobo Yin ${ }^{1}$, Weili Zhang ${ }^{2}$ \\ \& Xiang Zhang'
}

${ }^{1}$ NSF Nano-scale Science and Engineering Center (NSEC), 3112 Etcheverry Hall, University of California, Berkeley, California 94720, USA, ${ }^{2}$ School of Electrical and Computer Engineering, Oklahoma State University, Stillwater, Oklahoma 74078, USA, ${ }^{3}$ Huazhong University of Science and Technology, Wuhan, Hubei 430074, China, ${ }^{4}$ Departamento de Fisica Teorica de la Materia Condensada and Condensed Matter Physics Center (IFIMAC), Universidad Autonoma de Madrid, 28049 Madrid, Spain.

Spatial modulation of sound velocity below the wavelength scale can introduce strong frequency-dependent acoustic responses in tailored composite materials, regardless the fact that most natural bulk materials have negligible acoustic dispersions. Here, for the first time, we experimentally demonstrate a metamaterial that traps broadband acoustic waves and spatially separates different frequency components, as the result of dispersion and wave velocity control by designed gradient subwavelength structures. The trapping positions can be predicted by the microscopic picture of balanced interplay between the acoustic resonance inside individual apertures and the mutual coupling among them. With the enhanced wave-structure interactions and the tailored frequency responses, such metamaterial allows precise spatial-spectral control of acoustic waves and opens new venue for high performance acoustic wave sensing, filtering, and nondestructive metrology.

\footnotetext{
lowing down optical waves with resonating photonic structures introduces controllable optical delays and allows temporary storages of light ${ }^{1-5}$, resulting strong light-matter interactions lead to novel optical devices such as all-optical memories and switches. The causality, however, fundamentally restricts the attainable delay-bandwidth product, limiting the resonance-based slow-light applications to a narrow band of frequency. Adiabatic control of the dispersions lifts such a constraint and allows the recent demonstration of slow light device $^{6,7}$ and optical trapped rainbow ${ }^{8-15}$. In stark contrast to the tremendous progress in slow-light photonics, decelerating, trapping and spectrum-splitting of acoustic waves have not yet been realized due to the fact that nature occurring materials lack strong acoustic dispersion, which however is critical in many applications ranging from cochlear bionics to frequency mixing and selections in acoustic wave detection and imaging. Much like their optical counterparts such as photonic band-gap crystals and metamaterials ${ }^{16-18}$, artificial acoustic materials have shown promising potential of manipulating the acoustic wave and the wave-material interactions below the wavelength scale ${ }^{19-21}$. Innovative ways of acoustic wave collimation, focusing, cloaking, imaging, and extraordinary transmission have been explored ${ }^{22-27}$. However, strong enough acoustic dispersion that slows down and eventually leads to the trapping of sound waves with artificial materials has only been theoretically proposed ${ }^{28-30}$. By utilizing metamaterial design and tailoring its dispersion to an extreme level, here we experimentally demonstrate a new class of anisotropic metamaterials that can efficiently trap broadband acoustic waves and spatially split different frequency components. This effect is due to strong modulation of wave velocity through gradient subwavelength unit cells that are strongly coupled along the propagation direction. The decelerating process can be theoretically explained within the effective medium approach, which however tends to break at the trapping point. The accurate trapping locations are otherwise predicted by interpreting a balanced microscopic interplay solution, as we present in the following.
}

\section{Results}

The acoustic rainbow trapping metamaterials, as shown in Fig. 1a, consists of an array of grooves perforated on a rigid bar. The grooves, with linearly increasing depth $h$, have width $w$ and period $p$ that are much smaller than the wavelength of interest. This $2 \mathrm{D}$ material is described as an effective medium model of a tapered anisotropic metamaterial layer (medium I) sandwiched between the homogeneous and isotropic layers of environmental medium II and rigid body III (Fig. 1b). When considering an acoustic waves propagating along the metamaterial, we can analytically derive the corresponding group velocity $v_{g}$ of the fundamental guided mode from the general dispersion equation (see Supplementary Equation S8 online): 
a

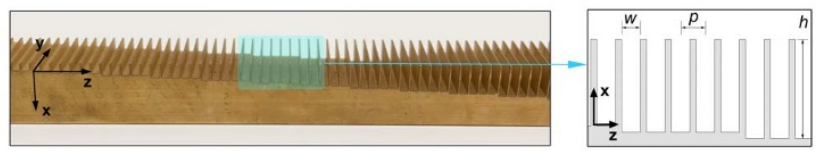

b

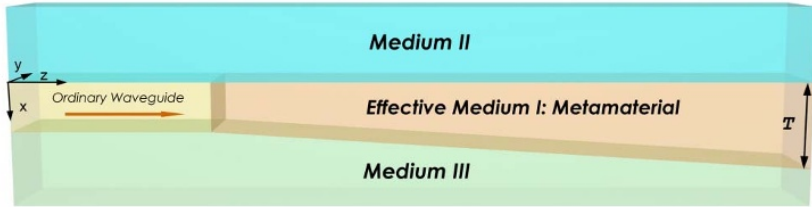

Figure 1 Metamaterial for acoustic rainbow trapping. (a) The metamaterial is made of 80 grooves perforated on a square brass alloy bar, with period $p=6.35 \mathrm{~mm}$ and width $w=4.76 \mathrm{~mm}$. 80 grooves are equally divided into 16 groups, which each contains 5 uniform grooves, while $h$ of each group increases from $1.59 \mathrm{~mm}$ to $25.4 \mathrm{~mm}$ with step of $1.59 \mathrm{~mm}$.

(b) Effective medium model of the composite structure consists of anisotropic metamaterial medium I sandwiched between two homogeneous and isotropic media II and III (respectively air and rigid material in this study). Orange arrow indicates wave propagation direction. In ideal two dimensional studies, the length of waveguide extends infinitely in the $y$ direction. Due to the limitation of fabrication technique, actual metamaterial extends $44.45 \mathrm{~mm}$, which is multiple times over largest wavelength of interest, in $y$ direction.

$$
v_{g}=\frac{c_{\text {air }}}{\sqrt{1+\left(\frac{\rho_{\text {air }}}{\rho_{x}} \tan \left(k_{0} \theta z\right)\right)^{2}}+k_{0} \theta z\left(\frac{\rho_{\text {air }}}{\rho_{x}}\right)^{2} \frac{\tan ^{2}\left(k_{0} \theta z\right)+1}{\sqrt{\cot ^{2}\left(k_{0} \theta z\right)+\left(\frac{\rho_{\text {air }}}{\rho_{x}}\right)^{2}}}},
$$

Here $k_{0}=\omega / c_{\text {air }}$ is the free space wave vector. $\theta$, as shown in Fig. $2 \mathrm{~b}$, is the tapering angle and $z$ is the distance along the $z$ axis. The effective anisotropic mass density and bulk modulus of metamaterial layer I are respectively $\rho=(p / w) \rho_{\text {air }}, \rho_{z}=\infty$ and $\kappa=(p / w) \kappa_{\text {air. }}$. As shown in Fig. 2a, calculated group velocity of the acoustic wave with given frequency $f$ progressively slows down and eventually stops at $z=c_{\text {air }} /(4 \theta f)$, as implied by equation (1). Such spatial evolution of acoustic wave group velocity is hardly found in nature materials, revealing the metamaterial's strong acoustic dispersive behavior, which is evidently shown in Fig. 2 b with significantly energy concentration of three different spectral components $(5 \mathrm{kHz}, 7 \mathrm{kHz}$ and $9 \mathrm{kHz}$ ) at various locations. This result is validated by the full wave 2D FEM simulation presented in Fig. 2c, suggesting that broadband incident acoustic waves can be trapped into spatially separated locations and therefore spectrally split, resembling the phenomenon of optical "trapped rainbow" ${ }^{8-15}$.

The experiments were conducted in air with a setup schematically shown in Fig. 3a. Figure 3b shows the position-dependent acoustic wave transmission along the metamaterial, which is measured at 4.5$9.5 \mathrm{kHz}$. Different frequency components are gradually stopped and successively localized along the waveguide, progressively inducing enhanced acoustic fields that approach the maximums. The intensities measured at the trapping point have over two orders of magnitude boost over non-trapping scenario, where the acoustic field is measured at the same distance from the speaker with reference metal bar containing uniform grooves. Such results are well agreed with the 3D full wave FEM simulation shown in Supplementary Fig. S2 online. The minor differences arise from the deviation that happens when simulation setup tries to accurately mimic the actual speaker configuration and ambient environment. The spatial evolutions of acoustic pressure fields for various frequencies $(4.5 \mathrm{kHz}, 6.0 \mathrm{kHz}$, and $9.5 \mathrm{kHz}$ ) are illustrated in Fig. 3c. Alike one can be concluded from the dispersion relation in Fig. $2 \mathrm{a}$ that is derived based on the effective medium model, higher frequency acoustic waves are clearly trapped in the region with shallower grooves, whereas lower frequency component like the $4.5 \mathrm{kHz}$ one propagates along the metamaterial until stops at section with deeper grooves. The experimental results agree well with full-wave 2D finite element method (FEM) simulation conclusion (Fig. 4b). It can be observed that the experimentally measured spectral splitting ( frequency $f$ versus trapped position $z$ ) exhibits a nonlinear distribution along the linearly tapered metamaterial, implying the non-uniform spatial density of the frequency components $\left|d f_{\text {stop }} / d z\right|=c_{a i r} /\left(4 \theta z^{2}\right)$, which can be derived from equation (1) and noticed in Fig. 2a. However, by tailoring the position-dependent groove depth gradient, arbitrary spatial separation of various spectral components can be realized.

To achieve the strong dispersion required for acoustic rainbow trapping, the groove fins of the metamaterial should be prevented to support any mode that could disturb the acoustic field in air. We have accordingly picked brass alloy as the base material, taking advantage of the modulus of rigidity about six orders of magnitude larger than that of air. Thus the influence of fin vibration is negligible when we experimentally study the fabricated metamaterial structure in solid-fluid system. We also conducted multiple measurements using the same setup. The results show small variations in acoustic pressure fields and trapping locations that result in the error bars in Fig. 3. Here it is worth noting that the demonstrated broadband acoustic wave decelerating, trapping, and spectral splitting do not rely on any phase manipulation along the propagation direction, very different from previously proposed acoustical cochlear-like response and place-to-frequency maps ${ }^{31-33}$.

The effective medium model can well describe the propagation and gradually slowing down of acoustic waves because of the deep subwavelength nature of unit cells in the metamaterial. However, when approaching the trapping location, the effective wavelength of acoustic wave is progressively decreased. Therefore these unit cells are no longer deep subwavelength in size, which leads to the breakdown of effective medium model, the deviation at predicting where the acoustic wave will be trapped and how the intensity of acoustic wave is distributed around the trapping location, as witnessed by different intensity peak positions and dropping rates observed in Fig. 2 and Fig 3. Under such circumstance, the mechanism of the acoustic wave localization at trapping point can be better interpreted by a microscopic model that the acoustic wave trapping originates from the balanced interplay between the acoustical oscillation inside individual grooves and the mutual near-field coupling among the neighboring grooves, as depicted in Fig. 4a. This coupling is governed by the diffracted evanescent waves with maximum momenta $\pm \pi / p$ that propagate along the interface between the metamaterial and the air, driving the pressures and particle velocity fields to oscillate in opposite phases in neighboring grooves, leading to a standing wave with diminishing group velocity at the trap. As a result, the accumulation of the acoustic energy and field enhancement is observed in Fig. 3. A closed-form-expression for this model can be derived (see supplementary information for details):

$$
\cot \left(k_{0} h\right)=\frac{k_{0} \sin \left(\frac{\pi}{p} \frac{w}{2}\right)}{\sqrt{\left(\frac{\pi}{p}\right)^{2}-k_{0}^{2}}},
$$

Here $\cot \left(k_{0} h\right)$ defines the acoustical oscillation localized within individual grooves, while the right side term is dominated by the diffracted evanescent waves, relating to the coupling effect discussed above. Balance between those two physical processes results in the trapped wave. Microscopically in Fig. 4a, it can also be seen as a series of closed-loop circulations of acoustic energy-flux between the adjacent grooves, resulting in the standstill of acoustic wave with zero group velocity. It's worth noting that for this microscopic model, only under the strict condition when the period of unit cells 


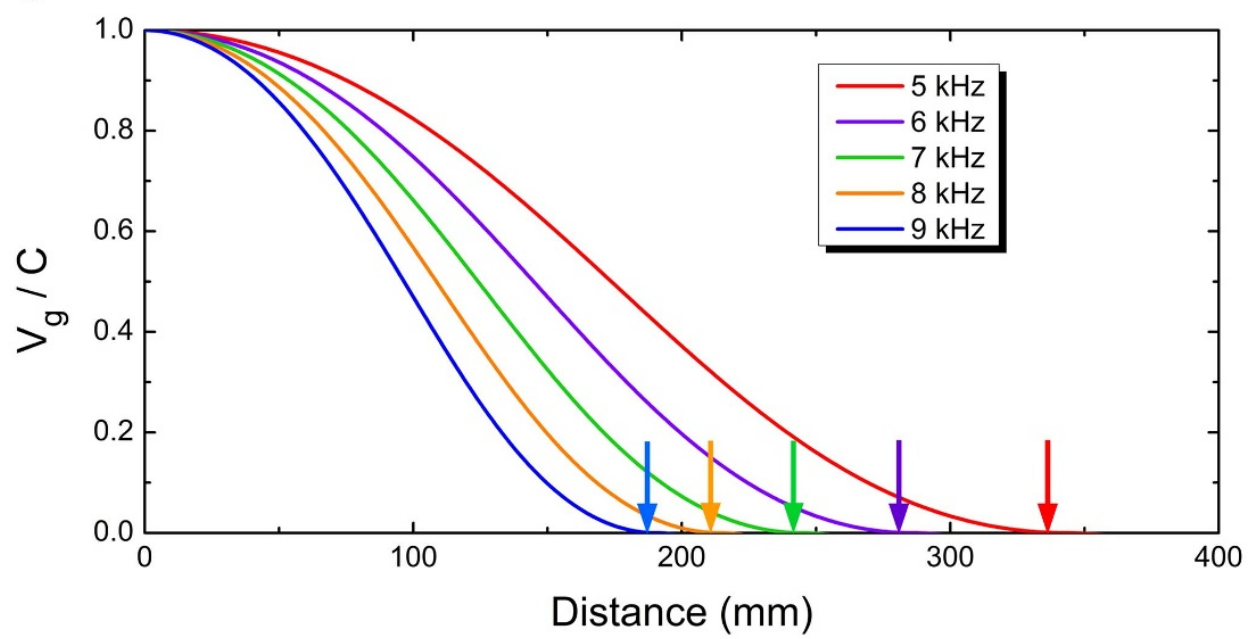

b
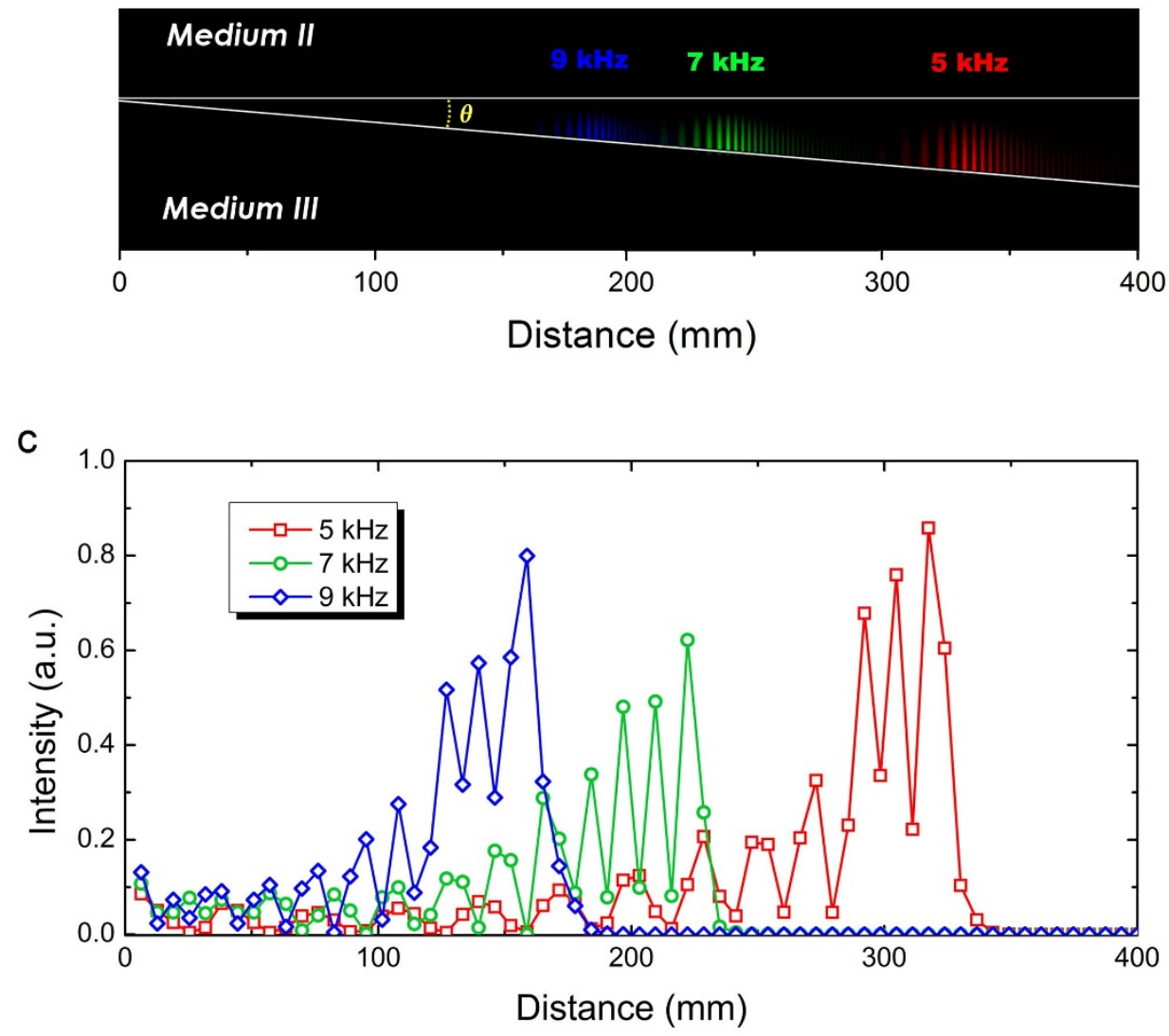

Figure 2 Spatial modulation of acoustic waves investigated within the effective medium approach and full wave simulation. (a) Calculated group velocities (normalized to the speed in air) of acoustic waves $(5 \mathrm{kHz}, 6 \mathrm{kHz}, 7 \mathrm{kHz}, 8 \mathrm{kHz}$ and $9 \mathrm{kHz}$, respectively) propagating along the metamaterials shown in Figure 1. Various spectral components are progressively slowed down and eventually trapped at different locations which are marked with colored arrows. The spatial density of trapped spectral components (space between the colored arrows) is non-uniform along the linearly tapered metamaterial. (b) Simulated spatial energy distribution of different acoustic waves ( $5 \mathrm{kHz}, 7 \mathrm{kHz}$, and $9 \mathrm{kHz}$ ), presented in RGB colors (Red, Green and Blue), shows significantly enhanced acoustic fields at the positions where different spectral components are trapped. $\theta$ is the tapering angle of metamaterials core. Simulation was carried out with partial differential equation module. The wave intensity is presented by the strength of each individual color. (c) Acoustic field intensity variation from full wave simulation on 2D actual structure reveals the same tendency of gradual field enhancement as the one derived from effective medium approach.

$\mathrm{p} \rightarrow 0$, there is an asymptote at $f_{\text {stop }}=c_{\text {air }} / 4 h$ derived from equation (2), and each groove can be treated as a quarter-wave resonator for trapped acoustic wave, which is consistent with equation (1) as perfect effective medium. However, due to the finite size of actual unit cells, the microscopic model is more properly suited to tell the exact acoustic wave trapping position and agrees better with the $2 \mathrm{D}$ fullwave FEM simulations on real metamaterial geometry than effective medium model, as presented in Fig. 4b. 
a

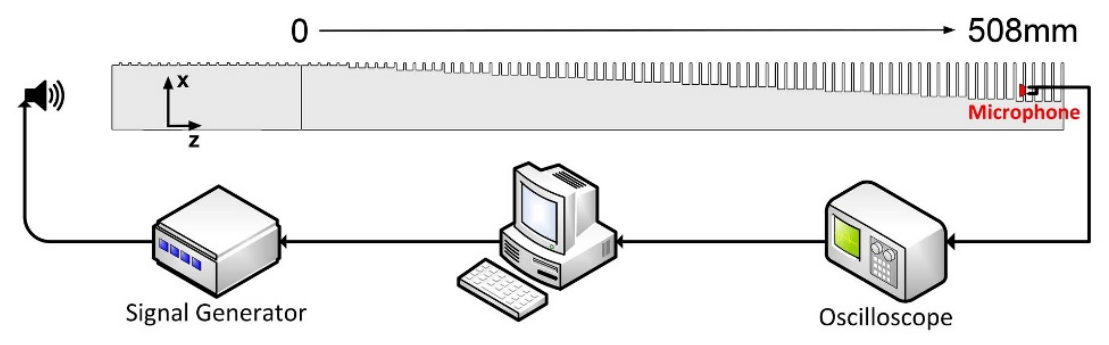

b

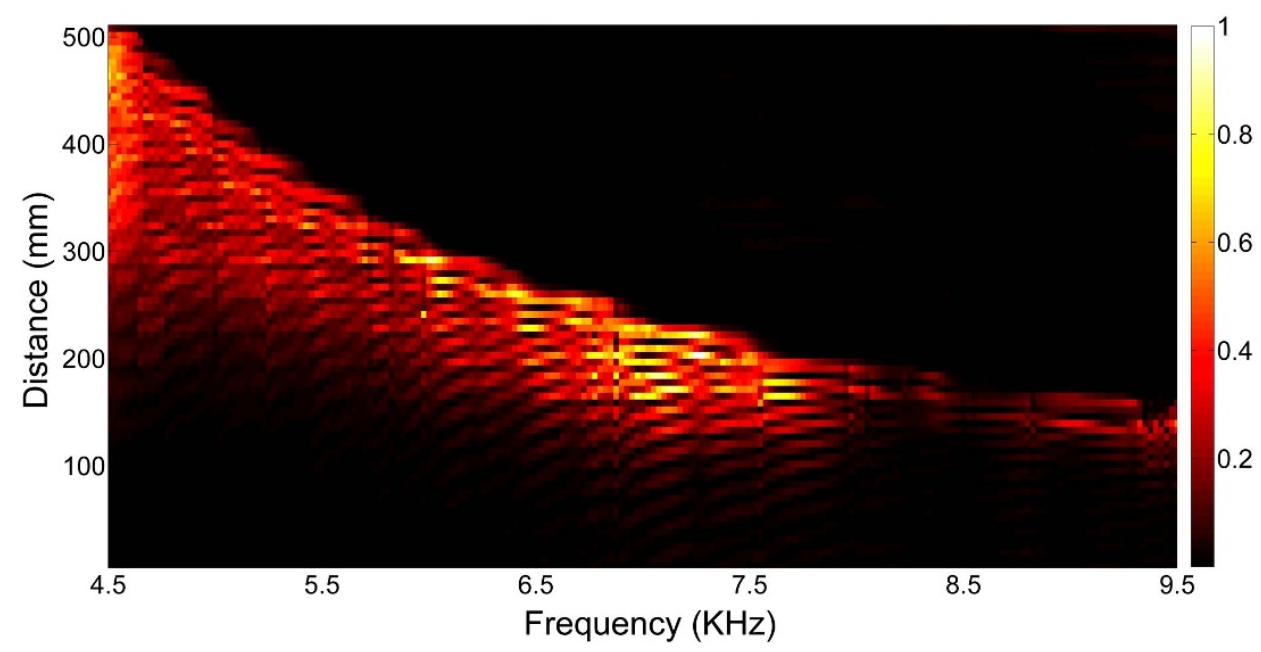

C

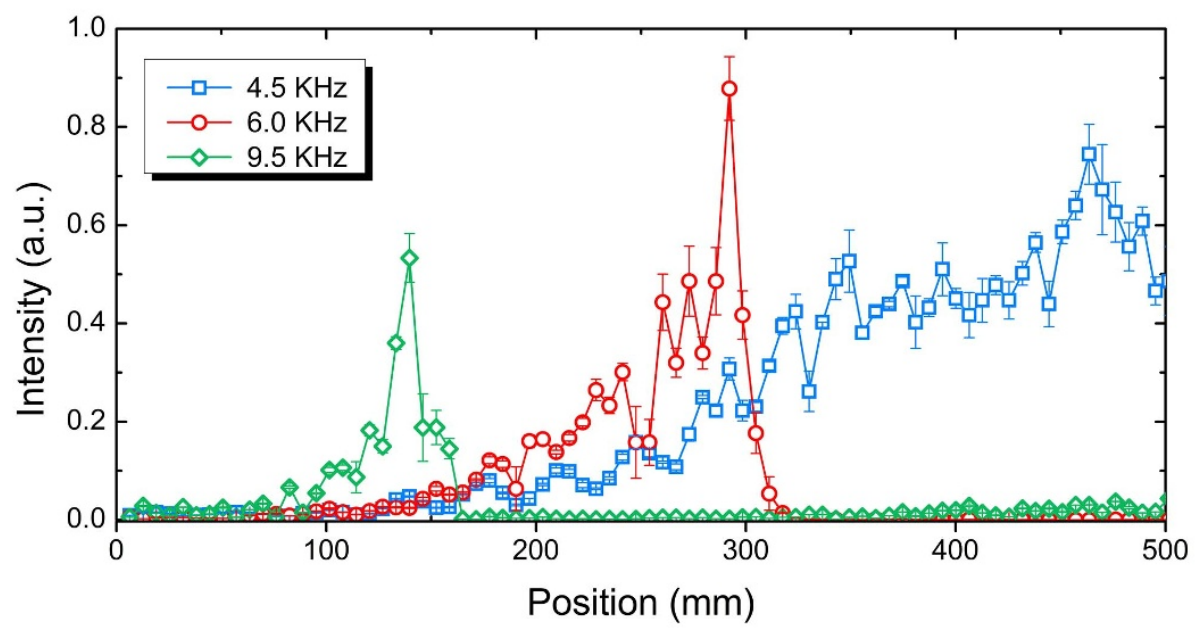

Figure 3 Experimental demonstration. (a) Schematic drawing of the experimental set-up. A guiding metal bar (dark Gray) is introduced to excite bound modes propagating along the surface, coupling acoustic waves towards the metamaterial sample. (b) Normalized acoustic fields intensity versus the measurement positions (distance from the start of metamaterial in $z$ direction) shows clear rainbow trapping effect, with incident waves changing from $4.5 \mathrm{kHz}$ to $9.5 \mathrm{kHz}$ by step of $25 \mathrm{~Hz}$. (c) Acoustic field intensity variation along the metamaterial for $4.5 \mathrm{kHz}, 6.0 \mathrm{kHz}$, and $9.5 \mathrm{kHz}$ incident waves. All three show progressively enhanced fields before peaking at spatially separated locations. The error bars represent s.d. among 5 repeat measurements.

\section{Discussion}

In summary, we have experimentally demonstrated that broadband acoustic waves can be gradually slowed down to standstill, thus trapped and spatial-spectrally separated when propagating along a metamaterial waveguide. Such "trapped rainbow" effect is achieved by the dispersive modulation of acoustic velocity through gradually varying subwavelength unit cells that are strongly coupled along the propagation direction. The acoustic "trapped rainbow" can be released by disrupting the balance between the acoustical oscillation inside individual grooves and the mutual near-field coupling among the neighboring grooves. A simple example is to place a small object at the top or inside trapping groove to strongly scatter otherwise trapped acoustic waves. Contrary to the optical counterparts ${ }^{34}$, this acoustic metamaterial with the unique spectral splitting capability is also suitable for 3D system due to the absence of cutoff wavelength. Without bandwidth limitation, it opens the door to realizing precise spatial-spectral control of acoustic waves. 


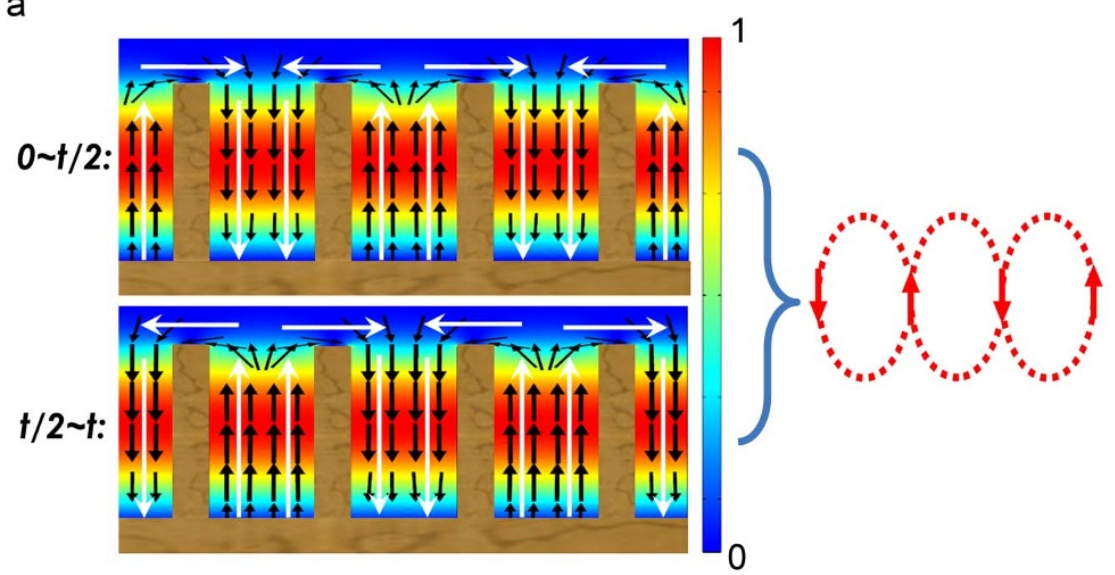

b

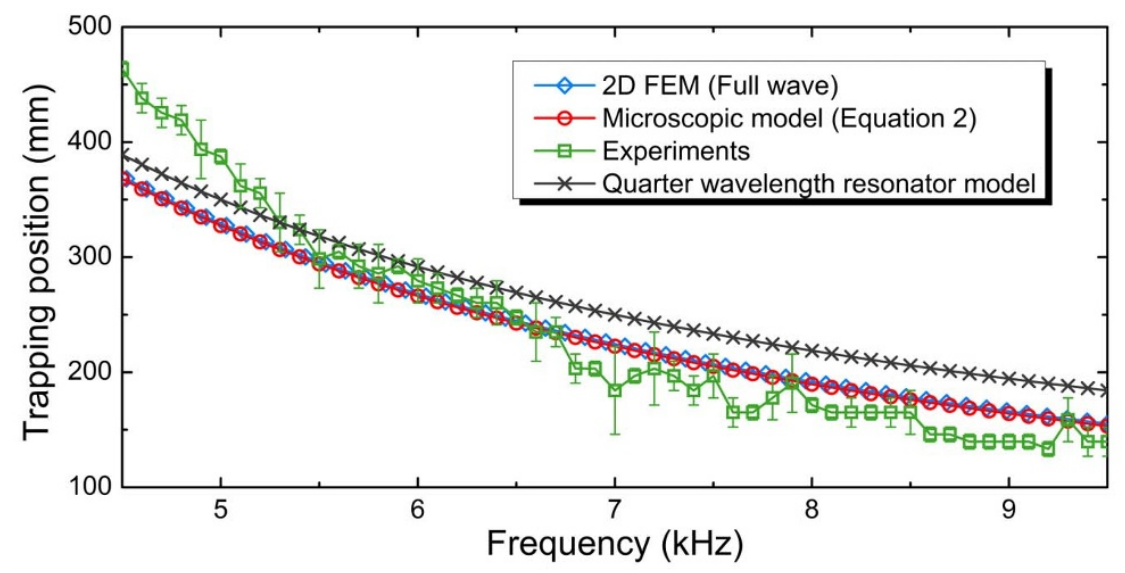

Figure $4 \mid$ Microscopic mechanism associated with steady acoustic wave trapping state. (a) Acoustic wave trapping is the result of balanced interplay between the local groove oscillation and the mutual near field coupling among the grooves to form closed-loop circulations of "trapped sound". When acoustic waves are trapped, the effective acoustic energy flow (black arrows) between two adjacent grooves will go opposite directions during 0 - $t / 2$ (top half) and $t / 2-t$ (bottom half), here $t$ is the period of oscillation inside individual groove, thus forming an equivalent closed-loop circulation where the acoustic wave always returns to its origin, resulting in the standstill of acoustic waves. The vertical white arrows represent acoustical oscillation inside the grooves. The horizontal white arrows correspond to the diffracted evanescent waves that propagate along the interface between the metamaterial and the air. The color map is the normalized acoustic energy flow. (b) Comparison of acoustic wave trapping locations derived by 2D full wave FEM simulation on actual metamaterial geometry, the microscopic model, the quarter-wavelength resonator model, and experimental results. Microscopic model result fits well with the FEM simulation curve and experimental results, suggesting better capability in analyzing the steady acoustic wave trapping state, especially when the unit cell size is not infinite small. Experimental results start to deviate from theoretical prediction at lower frequency range, which is due to the larger wavelength and finite length of actual sample in y direction. The error bars represent s.d. among 5 repeated measurements.

\section{Methods}

Sample preparation. We fabricated the metamaterial structure with a square brass alloy bar. 80 grooves are perforated on the bar by high pressure water jet cutting, with period $p=6.35 \mathrm{~mm}$ and width $w=4.76 \mathrm{~mm} .80$ grooves are equally divided into 16 groups, which each contains 5 uniform grooves, while $h$ of each group increases from $1.59 \mathrm{~mm}$ to $25.4 \mathrm{~mm}$ with step of $1.59 \mathrm{~mm}$.

Experiments setup and measurements. Experiments are conducted under room temperature inside a large chamber to isolate the environmental noise. Attached on all the interior walls, cone shaped sound absorbing foam resembles the perfect matching boundary around the setup. A square brass alloy bar with same width as the metamaterial and uniform shallow grooves, connected with the metamaterial at one side and acting as an ordinary input waveguide, couples the incident acoustic waves from the speaker into the metamaterial. A $17 \mathrm{~mm}$ diameter PUI Audio AST1732MR-R round speaker is located at the other side of input waveguide, sending out continuous sinusoidal waves towards the sample at various frequencies. The diameter of speaker is smaller than the shortest wavelength applied in test so that various spectral outputs possess no directivity difference. Incident signal were generated by Tektronix AFG3021B Arbitrary/Function generator. Acoustic pressure fields are measured by inserting a $4 \mathrm{~mm}$ diameter PUI Audio TOM-1045S-C33-R microphone from the side into each of the 80 grooves and close to the bottom. This microphone is attached on a thin metal wire to minimize field perturbations. All measured data was sent to computer through Tektronix TDS2002B digital storage oscilloscope for further processing. Both AFG3021B and TDS2002B were controlled by LabVIEW ${ }^{\circledR}$ software.

Numerical calculations. FEM simulations on acoustic pressure field measurement were carried by COMSOL Multiphysics ${ }^{\mathrm{TM}} 4.1$ with the acoustic-solid interaction multi-physics module. The materials applied in simulations were air and ultramachinable brass (Alloy 360). Plane wave radiation boundary conditions were given to the outer boundaries of simulation domain so there will be no interference from reflected acoustic wave. The largest mesh element size was set lower than $1 / 10$ of the lowest wavelength, and finer meshes were applied at the domain of metal fins.

1. Noda, S., Chutinan, A. \& Imada, M. Trapping and emission of photons by a single defect in a photonic bandgap structure. Nature 407, 608-610 (2000).

2. Julsgaard, B., Sherson, J., Cirac, J. I., Fiurásek, J. \& Polzik, E. S. Experimental demonstration of quantum memory for light. Nature 432, 482-486 (2004).

3. Xia, F., Sekaric, L. \& Vlasov, Y. Ultracompact optical buffers on a silicon chip. Nature Photonics 1, 65-71 (2007). 
4. Yanik, M. F. \& Fan, S. H. Dynamic photon storage. Nature Physics 3, 372-374 (2007).

5. Fiore, V. et al. Storing optical information as a mechanical excitation in a silica optomechanical resonator. Physical Review Letters 107, 133601 (2011).

6. Yanik, M. F. \& Fan, S. Stopping light all optically. Physical Review Letters 92 083901 (2004).

7. Xu, Q., Dong, D. \& Lipson, M. Breaking the delay-bandwidth limit in a photonic structure. Nature Physics 3, 406-410 (2007)

8. Tsakmakidis, K. L., Boardman, A. D. \& Hess, O. “Trapped rainbow" storage of light in metamaterials. Nature 450, 397-401 (2007).

9. Gan, Q., Ding, Y. \& Bartoli, F. "Rainbow" trapping and releasing at telecommunication wavelengths. Physical Review Letters 102, 056801 (2009).

10. Williams, C. R. et al. Highly confined guiding of terahertz surface plasmon polaritons on structured metal surfaces. Nature Photonics 2, 175-179 (2008).

11. Gan, Q. et al. Experimental verification of the rainbow trapping effect in adiabatic plasmonic gratings. Proceedings of the National Academy of Sciences of the United States of America 108, 5169 (2011).

12. Jang, M. \& Atwater, H. Plasmonic rainbow trapping structures for light localization and spectrum splitting. Physical Review Letters 107, 207401 (2011).

13. Kats, M. A., Woolf, D., Blanchard, R., Yu, N. \& Capasso, F. Spoof plasmon analogue of metal-insulator-metal waveguides. Optics Express 19, 16, 14860-14870 (2011)

14. Chen, L., Wang, G. P., Gan, Q. \& Bartoli, F. J. Trapping of surface-plasmon polaritons in a graded Bragg structure: Frequency-dependent spatially separated localization of the visible spectrum modes. Physical Review B 80, 161106 (2009).

15. He, S., He, Y. \& Jin, Y. Revealing the truth about 'trapped rainbow' storage of light in metamaterials. Scientific Reports 2, 583 (2012).

16. Smith, D., Padilla, W., Vier, D., Nemat-Nasser, S. \& Schultz, S. Composite medium with simultaneously negative permeability and permittivity. Physical Review Letters 84, 4184 (2000).

17. Shelby, R., Smith, D. \& Schultz, S. Experimental verification of a negative index of refraction. Science 292, 77 (2001).

18. Dolling, G., Enkrich, C., Wegener, M., Soukoulis, C. M. \& Linden, S. Simultaneous negative phase and group velocity of light in a metamaterial. Science 312, 892-4 (2006).

19. Liu, Z. et al. Locally resonant sonic materials. Science 289, 1734-1736 (2000).

20. Fang, N. et al. Ultrasonic metamaterials with negative modulus. Nature Materials 5, 452-6 (2006).

21. Ding, Y., Liu, Z., Qiu, C. \& Shi, J. Metamaterial with simultaneously negative bulk modulus and mass density. Physical Review Letters 99, 093904 (2007).

22. Chen, H. \& Chan, C. T. Acoustic cloaking in three dimensions using acoustic metamaterials. Applied Physics Letters 91, 183518 (2007).

23. Lu, M. H. et al. Extraordinary acoustic transmission through a $1 \mathrm{~d}$ grating with very narrow apertures. Physical Review Letters 99, 174301 (2007)

24. Zhu, X. F., Liang, B., Kan, W. W., Zou, X. Y. \& Cheng, J. C. Acoustic cloaking by a superlens with single-negative materials. Physical Review Letters 106, 014301 (2011).

25. Zhang, S., Yin, L. \& Fang, N. Focusing ultrasound with an acoustic metamaterial network. Physical Review Letters 102, 194301 (2009).
26. Li, J., Fok, L., Yin, X., Bartal, G. \& Zhang, X. Experimental demonstration of an acoustic magnifying hyperlens. Nature Materials 8, 931-4 (2009).

27. Christensen, J., Fernandez-Dominguez, A. I., de Leon-Perez, F., Martin-Moreno, L. \& Garcia-Vidal, F. J. Collimation of sound assisted by acoustic surface waves. Nature Physics 3, 851-852 (2007).

28. Christensen, J., Huidobro, P. A., Martín-Moreno, L. \& García-Vidal, F. J. Confining and slowing airborne sound with a corrugated metawire. Applied Physics Letters 93, 083502 (2008).

29. Mciver, M. Acoustic Wave trapping in one-dimensional axisymmetric arrays. The Quarterly Journal of Mechanics and Applied Mathematics 64, 401-414 (2011)

30. Liang, Z. \& Li, J. Extreme acoustic metamaterial by coiling up space. Physical Review Letters 108, 114301 (2012).

31. White, R. D. \& Grosh, K. Microengineered hydromechanical cochlear model. Proceedings of the National Academy of Sciences of the United States of America 102, 1296-301 (2005).

32. Mayon $\mathrm{Ku}, \mathrm{E}$. Modelling the human cochlea. The Journal of the Acoustical Society of America 126, 3373 (2009).

33. Martignoli, S. \& Stoop, R. Local cochlear correlations of perceived pitch. Physical Review Letters 105, 048101 (2010).

34. Pendry, J. B., Martin-Moreno, L. \& Garcia-Vidal, F. J. Mimicking Surface Plasmons with Structured Surfaces. Science 305, 847-848 (2004).

\section{Acknowledgements}

J.Z., X.F.Z., X.Y. and X.Z. acknowledge financial support from the NSF Nano-scale Science and Engineering Center (NSEC) for Scalable and Integrated Nanomanufacturing (SINAM) (CMMI-0751621). Y.C., W.Z. acknowledge financial support from the NSF

(ECCS-1232081) and the National Science Foundation of China (grant no. 61028011).

F.J.G.-V. acknowledges financial support from the Spanish MINECO under contract MAT2011-28581-C02-01.

\section{Author contributions}

J.Z., X.F.Z., X.Y. and X.Z. designed and conducted the experiment. Y.C., J.Z., X.F.Z., F.J.G.-V. and W.Z. performed analytical and numerical computations. X.Z. and W.Z. conceived and led the project. All authors contributed to the manuscript writing and revision.

\section{Additional information}

Supplementary information accompanies this paper at http://www.nature.com/ scientificreports

Competing financial interests: The authors declare no competing financial interests.

License: This work is licensed under a Creative Commons

Attribution-NonCommercial-NoDerivs 3.0 Unported License. To view a copy of this license, visit http://creativecommons.org/licenses/by-nc-nd/3.0/

How to cite this article: Zhu, J. et al. Acoustic rainbow trapping. Sci. Rep. 3, 1728; DOI:10.1038/srep01728 (2013) 